\title{
Assessing the quality of inspection for tapered aircraft fastener holes using an engineer's blue contact test
}

DOI:

10.1016/j.precisioneng.2020.01.004

\section{Document Version}

Accepted author manuscript

Link to publication record in Manchester Research Explorer

\section{Citation for published version (APA):}

Lawless, B., Heinemann, R., \& Liaqat, A. (2020). Assessing the quality of inspection for tapered aircraft fastener holes using an engineer's blue contact test. Precision Engineering, 63, 62-67.

https://doi.org/10.1016/j.precisioneng.2020.01.004

\section{Published in:}

Precision Engineering

\section{Citing this paper}

Please note that where the full-text provided on Manchester Research Explorer is the Author Accepted Manuscript or Proof version this may differ from the final Published version. If citing, it is advised that you check and use the publisher's definitive version.

\section{General rights}

Copyright and moral rights for the publications made accessible in the Research Explorer are retained by the authors and/or other copyright owners and it is a condition of accessing publications that users recognise and abide by the legal requirements associated with these rights.

\section{Takedown policy}

If you believe that this document breaches copyright please refer to the University of Manchester's Takedown Procedures [http://man.ac.uk/04Y6Bo] or contact uml.scholarlycommunications@manchester.ac.uk providing relevant details, so we can investigate your claim.

\section{OPEN ACCESS}




\title{
Assessing the quality of inspection for tapered aircraft fastener holes using an engineer's blue contact test
}

\author{
Becan Lawless 1, a, Robert Heinemann 1, b, Amer Liaqat 2, c \\ 1 The University of Manchester, Department of Mechanical, Aerospace and Civil \\ Engineering, Sackville Street, Manchester, M1 3BB, UK \\ 2 Airbus Operations Limited, Chester Road, Broughton, Chester, CH4 ODR, UK \\ a becan.lawless@postgrad.manchester.ac.uk, brobert.heinemann@manchester.ac.uk, c \\ amer.liaqat@airbus.com
}

Keywords: Aircraft fasteners, Taper-lok inspection, engineer's blue, blue check

\begin{abstract}
$\underline{\text { Abstract }}$
Tapered interference-fit fasteners (e.g. Taper-lok) are commonly used by the aerospace industry due to their high fatigue strength. The inspection relies on a 'true' geometry gauge coated with a thin film of engineer's blue being brought firmly into contact with the tapered hole. The pattern created on the gauge is then used to identify bearing surface area and distribution. This research investigates if the inspection reflects the hole geometry, and under what conditions the inspection may be assumed valid. The results show that the shade of the blue on the gauge as inspected through computer vision correlates with the unloaded geometry evaluated via a roundness tester, in particular when the red channel is taken alone. The inspection method falls short of accurately representing small features, particularly those tangential to the gauge removal direction. Thresholding visually or by computer vision may reasonably be used to identify the contact region pattern, however factors limiting blue migration mean the contact area may appear smaller than it actually is.
\end{abstract}

\section{Introduction}

In cases where fatigue loading is considered a priority, modern aircraft often use tapered shank bolts, referred to in industry as Taper-lok bolts. Relying on interference fit for fatigue strength (Salter, et al., 1966), the level of contact between bolt and hole, produced using reaming, is generally taken to be indicative of their installation quality and expected performance (Molent, 2010). A poorly installed Taper-lok was deemed central in the premature failure of an F-111 wing failing unexpectedly during fatigue testing (Harding, et al., 2006). Failure originated at a Taper-lok fastener which, along with many other tapered fasteners, was deemed to have a poor surface finish and insufficient interference to contribute any fatigue benefit (Clark, 2005). Thus aircraft manufacturers specify minimum interference levels for full load transfer between fastener and structure and to mitigate fretting (Tsoi \& 
Rajic, 2008). A similar emphasis is put on fretting and corrosion from ingress of contaminants as primary mechanisms for wear and eventual failure in prosthetic hip tapered interference-fit joints (Affatato \& Ruggiero, 2019).

Patent documentation describes inspection involving a bearing check pin with an ideal taper and thin film of engineer's blue being brought firmly into contact with the subject female surface via a slide hammer. A pattern is created at the interface, which is maintained assuming clean removal along the central axis. This engineer's blue pattern is used to determine the bearing surface quantity and distribution since firm contact will force the substance away resulting in lighter shades of blue. A good hole is generally characterised by a radial pattern of concentric rings along the shaft length corresponding to machining marks from tool rotation (Lambert, 1974).

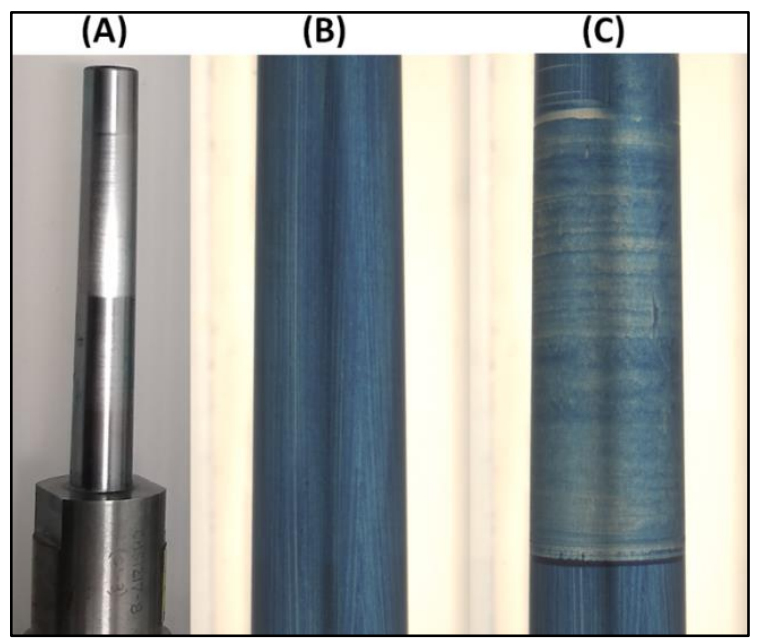

Figure 1: Clean tapered check pin (A), with engineer's blue layer applied by gloved finger (B) and pin surface displaying good contact pattern after impacting female subject bore (C)

Orthopaedic hip replacement research presents a method for evaluating wear of spherical heads and tapered interference connections for hip prosthetics. The surface geometry of a used prosthetic femoral head was measured via a coordinate measurement machine (CMM), and then pre-worn geometry assessed using a best-fit calculation to plot a surface. The furthest points were disregarded until a stable solution presented (Morlock, et al., 2006). This method has also been used in accessing wear on the tapered connection for hip-resurfacing modular heads, both cylindrical taper (Bishop, et al., 2013), and non-cylindrical taper (Buente, et al., 2015). Wear assessment data can be presented as a rainbow colour ramp 3D $\mathrm{XYZ}$ plot, and as a 2D polar plot of $\mathrm{Z}$ and theta, by ignoring the $\mathrm{r}$ component (Martin, et al., 2018). To acquire topography of greater detail researchers have also used dedicated roundness inspection instruments, e.g. Talyrond 365. (Matthies, et al., 2013) (Hothi, et al., 
2013) (Bills, et al., 2015) (Bills, et al., 2017) (Hothi, et al., 2017). A similar method of least squares definition of a best-fit cone has also been used to evaluate conicality error for quality evaluation (He, et al., 2014).

Currently there is little to no scientific literature on inspecting Taper-lok fasteners. Their inspection, while common practice, is effectively unresearched and has remained unchanged since the 1970s. This research aims to evaluate the effectiveness of engineer's blue inspection as a means to assess factors relating to Taper-lok performance. This further makes comparison to future inspections possible by establishing a baseline for the current system.

\section{$\underline{\text { Data collection }}$}

In order to assess the inspection quality of the blue check method, this research aimed to develop a comparison methodology between a "true" geometry measurement captured with a contact roundness machine, and an engineer's blue-covered gauge imprint. As described earlier, this involved the placement of the blue covered gauge in the tapered borehole, and axial striking via slide hammer to create contact patterns through local surface forces. A reverse strike of the slide hammer was used to remove the gauge axially without damage to the imprint. Using a methodology inspired by prosthetic wear research, physical geometries were taken in reference to a best-fit cone.

Since the gauge surface is a shallow angled cone, a single conventional photograph is insufficient. A bespoke lighting array with diffuse illumination was used to remove reflections then a compilation image was created to provide a $2 \mathrm{D}$ representation of the entire surface contact region. The three resulting compilation images are illustrated in Figure 2.

(A)

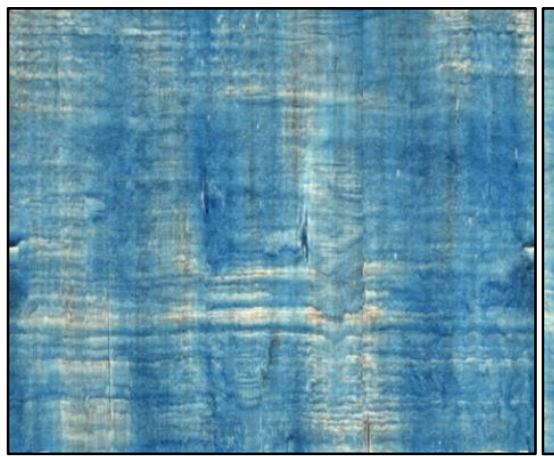

(B)

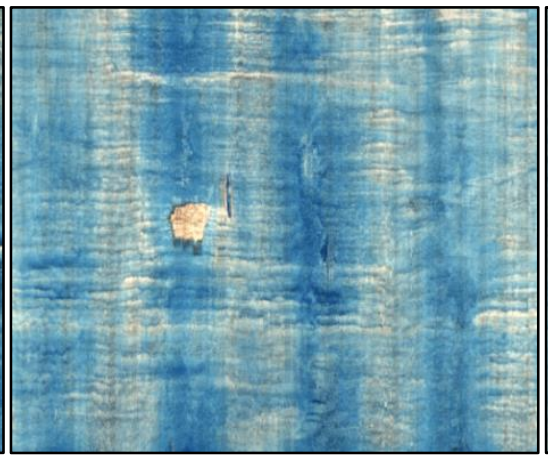

(C)

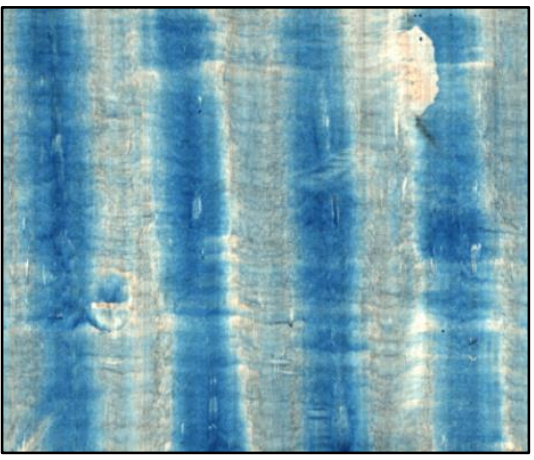

Figure 2: Compiled image from Taper-lok blue check representing full $360^{\circ}$ of gauge surface for holes with 'Good' (A), 'Medium' (B) and 'Bad' (C) quality 
The identification of these images' quality levels is based on the understanding that regular horizontal marks along an entire width denote areas in full supported contact with the gauge, and strong vertical colour separation denotes contact with poor conicality such that the surface is supported only along four light vertical bands. It is considered that these vertical bands can disrupt the stress patterns in the interference fit reducing the fasteners quality, and may in extreme cases act as leak paths for chemical ingress enabling corrosion. The process for creating the unwrapped images is shown in Figure 3.

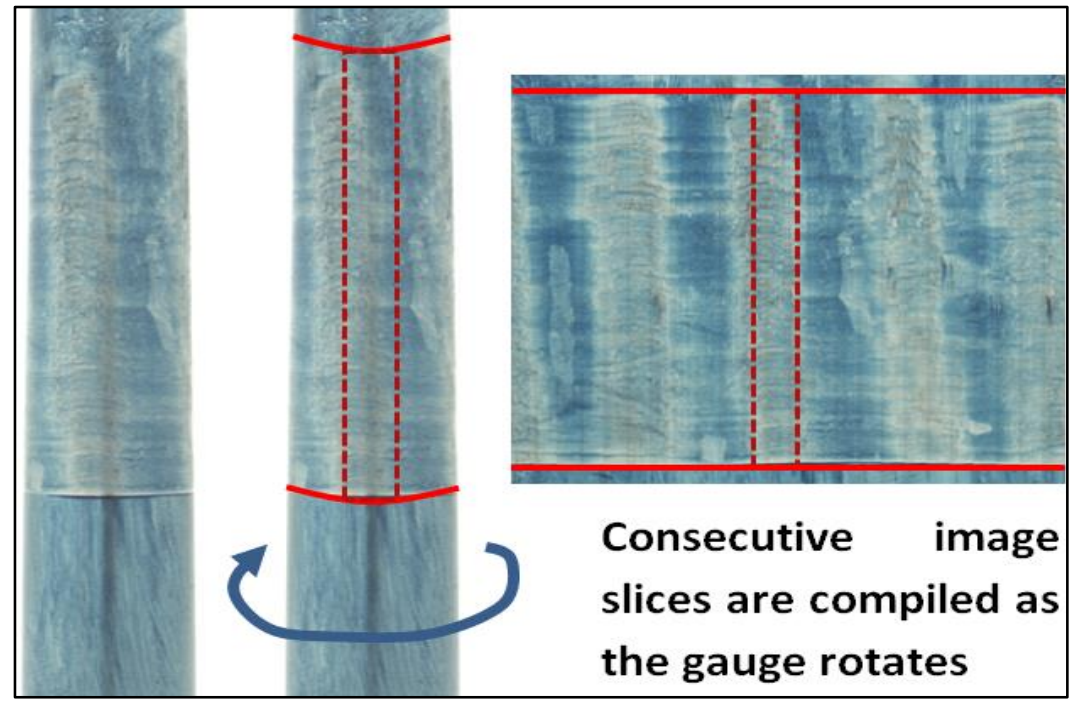

Figure 3: Visual illustration of method for creating a representative 'unwrapped' view of a $360^{\circ}$ conical surface from compiled images at set rotation increments

Following collection of Figure 2 imprint images samples were separated from the large coupon using wire EDM to $\varnothing 22 \mathrm{~mm}$, the top diameter of the bore's countersink shown in Figure 4 (left). The position of the central axis was registered based off the conical countersink using a coordinate measuring machine (CMM). The surfaces of three samples, and the conical gauge, were then registered using a Hommel-Etamic Roundscan 535 as shown in Figure 4 (right). This system automatically ensured its output polar displacement data was correctly axially centred. The system used a $0.3 \mathrm{~mm}$ radius stylus tip, and was calibrated to a repeatability of $0.09 \mu \mathrm{m}$.

While some methods identified in the literature fitted through regions considered unworn, this was inapplicable as the assessment considers manufacturing error from machining so there was no "unworn" equivalent. Instead the cone angle and minimum radius were optimised via a linear-regression ignoring the angle component from the polar data. Thus these two factors were optimised to minimise the root-mean-squared-error (RMSE) between the data and ideal projected cone over the entire dataset. 


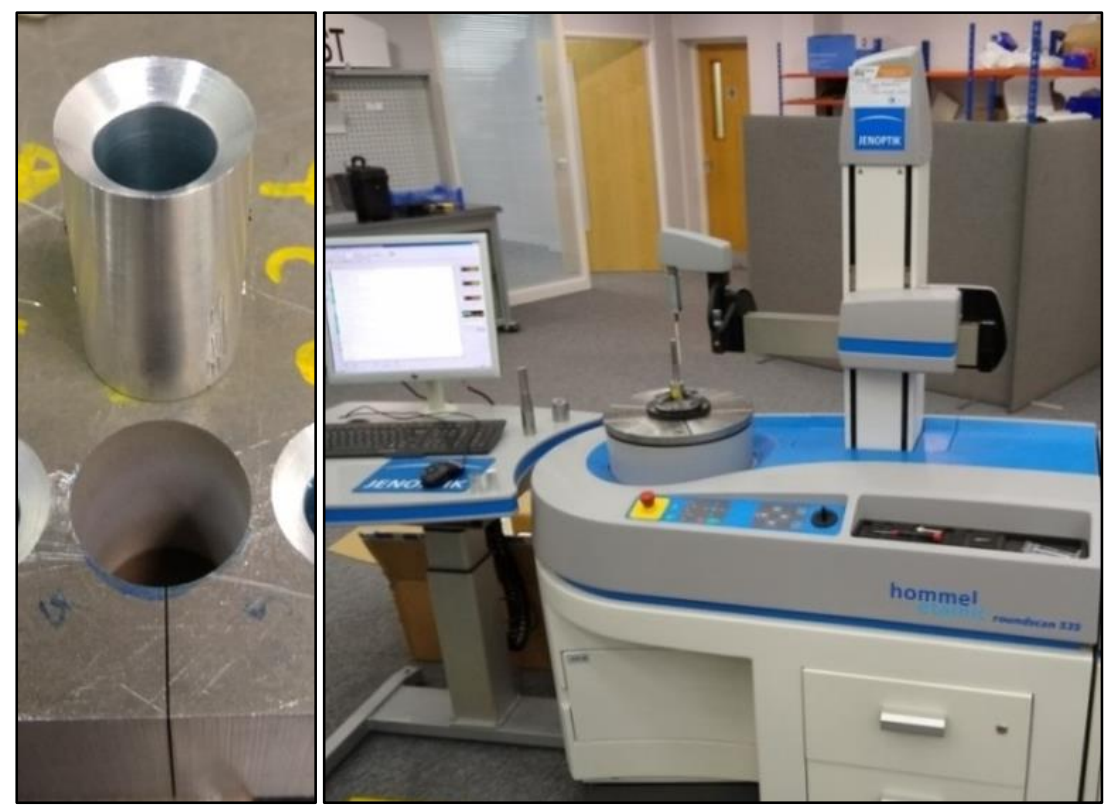

Figure 4: Wire EDM removed sample from aluminium coupon of tapered holes (left) and installed in Hommel-Etamic Roundscan $\mathbf{5 3 5}$ for measurement (right)

Using this best-fit cone for each measurement point a displacement from ideal was acquired.

Displacement was taken horizontally along the $\mathrm{R}$ axis, as opposed to normally from the surface, but with the shallow cone angle the displacement discrepancy was in the order of thousandths of a percent. Fitting the data in this way provides both positive and negative displacement values, meaning the cone does not necessarily reflect the actual bearing surface position. However since the overall conicality is sufficiently low it is likely that the actual surface would rest parallel inside this cone. These conicalities were respectively $21.6 \mu \mathrm{m}$, $34.3 \mu \mathrm{m}$, and $31.1 \mu \mathrm{m}$ for 'Good', 'Medium' and 'Bad' quality bore holes. By taking the third standard deviation and truncating the top and bottom $0.151 \%$ to remove outliers these values reduced to $11.0 \mu \mathrm{m}, 12.9 \mu \mathrm{m}$ and $20.2 \mu \mathrm{m}$.

An example displacement from best-fit cone in 3D Cartesian space can be seen in Figure 5. The subsequent colour-maps in 2D are shown in Figure 6. They are derived from polar coordinate space where the $\mathrm{x}$-axis denotes the data taken at each radial height each approximately $0.09^{\circ}$ apart, resulting in 4000 equally spaced measurement points, and the $y-$ axis denotes the 348 radial passes, each $0.1 \mathrm{~mm}$ apart. All three can be seen to have two identically spaced scratches resulting from unintended manufacturing errors. These however proved to be useful in confirming the alignment between scanned data and image. 


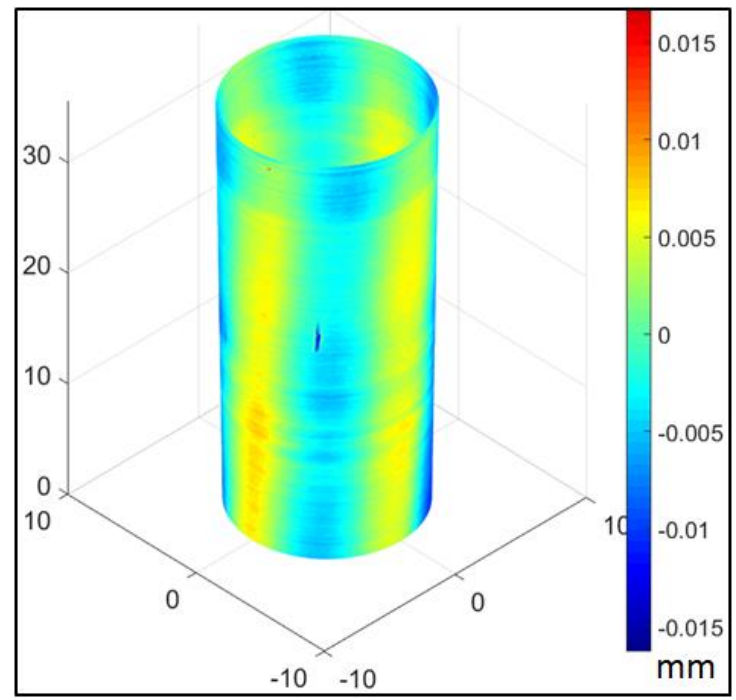

Figure 5: 3D representation of measured points from Roundscan data with displacement colour map showing displacement from best-fit cone for 'Bad' quality hole
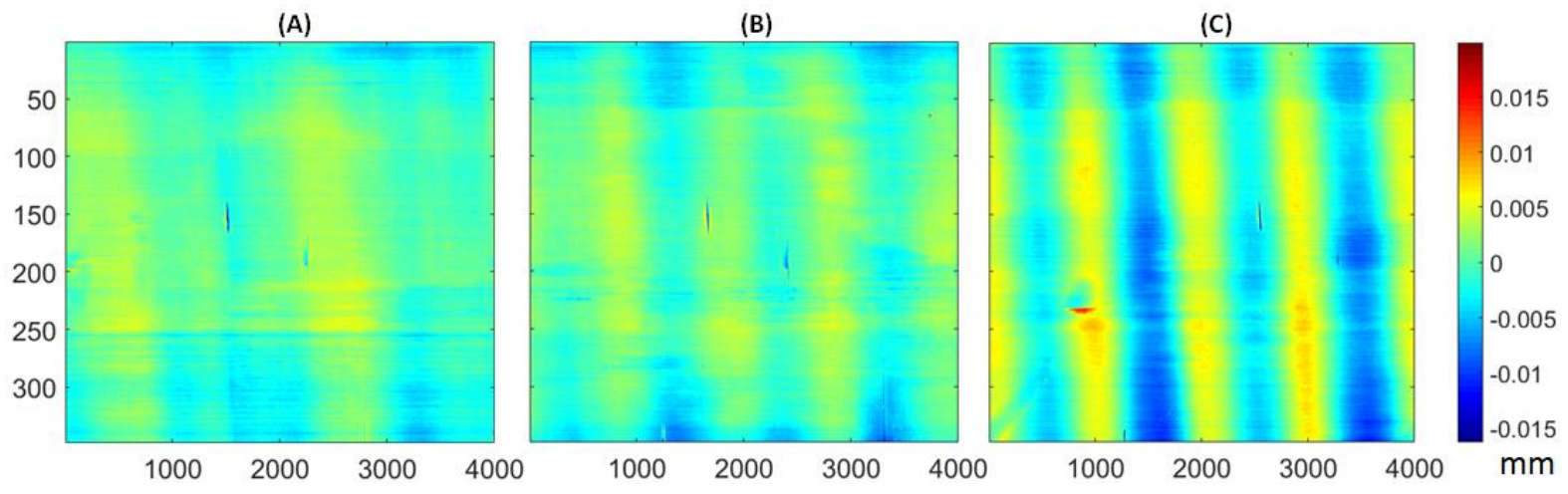

Figure 6: Radial displacement in polar space from best-fit cone for holes with 'Good' (A), 'Medium' (B) and 'Bad' (C)

quality

In order to validate the gauge's use as a 'true' surface it was also scanned using the same method described above to test its conicality over the region of expected contact plus a $1 \mathrm{~mm}$ buffer. The total range of displacements was $6.9 \mu \mathrm{m}$ however this includes what appears to be a speck of dust. By taking the third standard deviation and truncating the top and bottom $0.15 \backslash \%$ to remove outliers this value drops to $2.8 \mu \mathrm{m}$.

\section{Experimental results - Validity of shade inspection}

In order to make a reasonable direct comparison between the data sets in Figures 2 and 6 their dimensions had to be unified. In order to achieve this meaningfully some area averaging was required to mitigate minor alignment issues. As such both sets were reduced to an averaged 32 × 32 grid. The grid size presented a balancing issue with a coarser grid increasing comparative accuracy but with fewer data points to make a comparison. Having unified the data sets with averaged regions direct comparison could be made between the 
greyscale pixel intensity and corresponding displacements from the ideal cone. Pixel value was selected as an independent variable to represent the optical aspect of the current inspection. It is currently assumed an inspector can visually assess the contact levels via the shades of blue on the gauge. For this reason a computer vision assessment of the shade is used to stand in for the human in a more repeatable manner.

In making the comparison three regions were identified as smear points where the engineer's blue had rubbed away entirely as a result of the gauge's removal illustrated in Figure 7. These smear point regions were isolated due to their non-representative nature. The primary variable to be used in assessing the effectiveness of this inspection method is the R-squared value for each of the three samples. The comparisons between the two data-types are presented as scatter plots in Figure $\mathbf{8}$ with the smear points isolated in red.

(A)

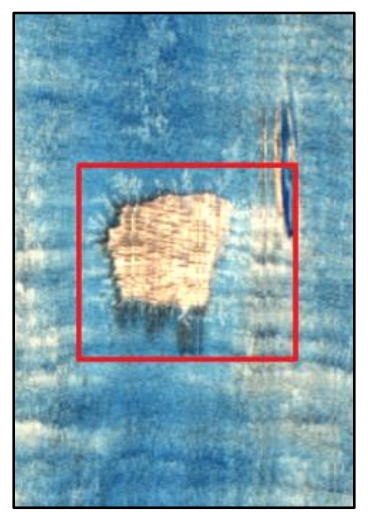

(B)

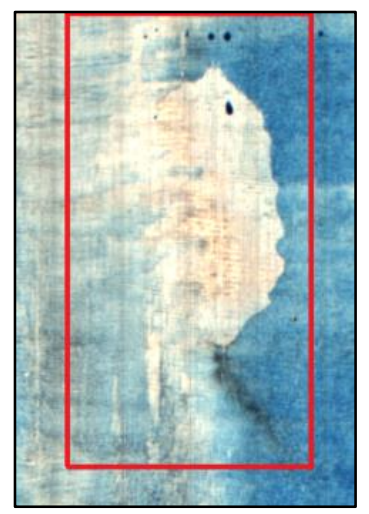

(C)

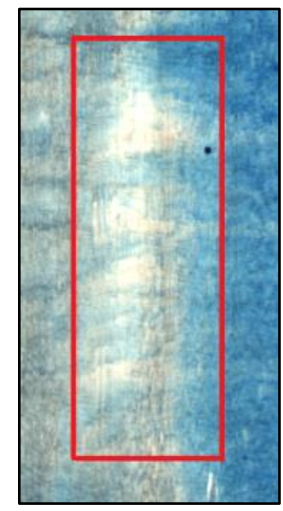

Figure 7: Smear point from 'Medium' quality hole (A), 'Bad' quality hole (B), and a second from the 'Bad' hole (C)

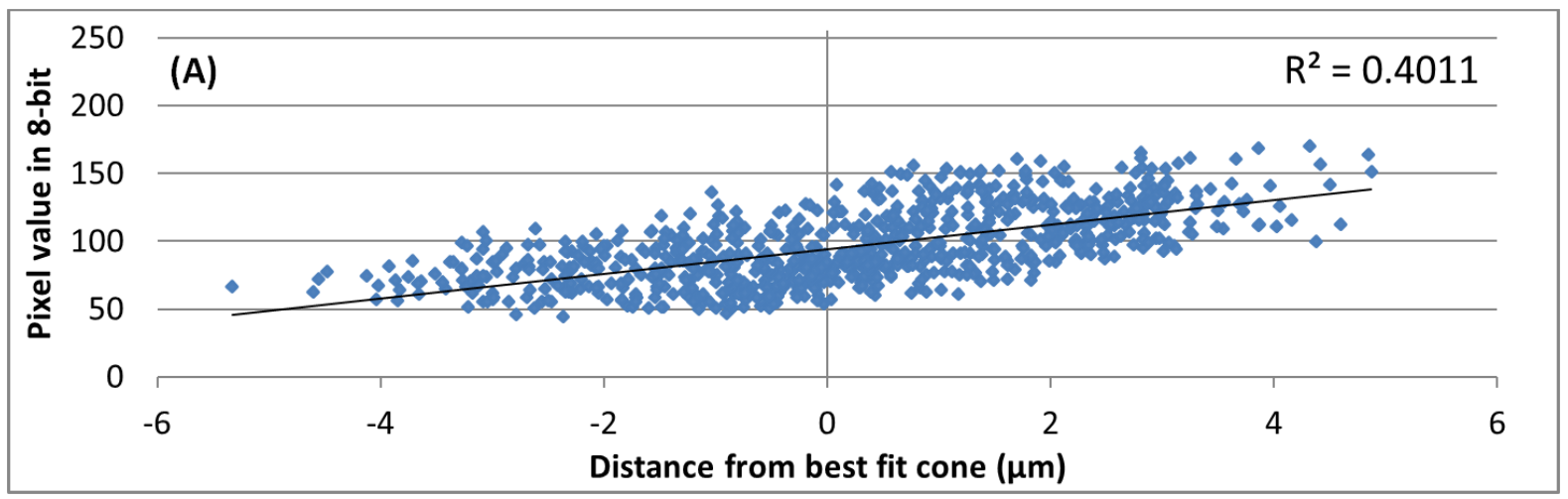




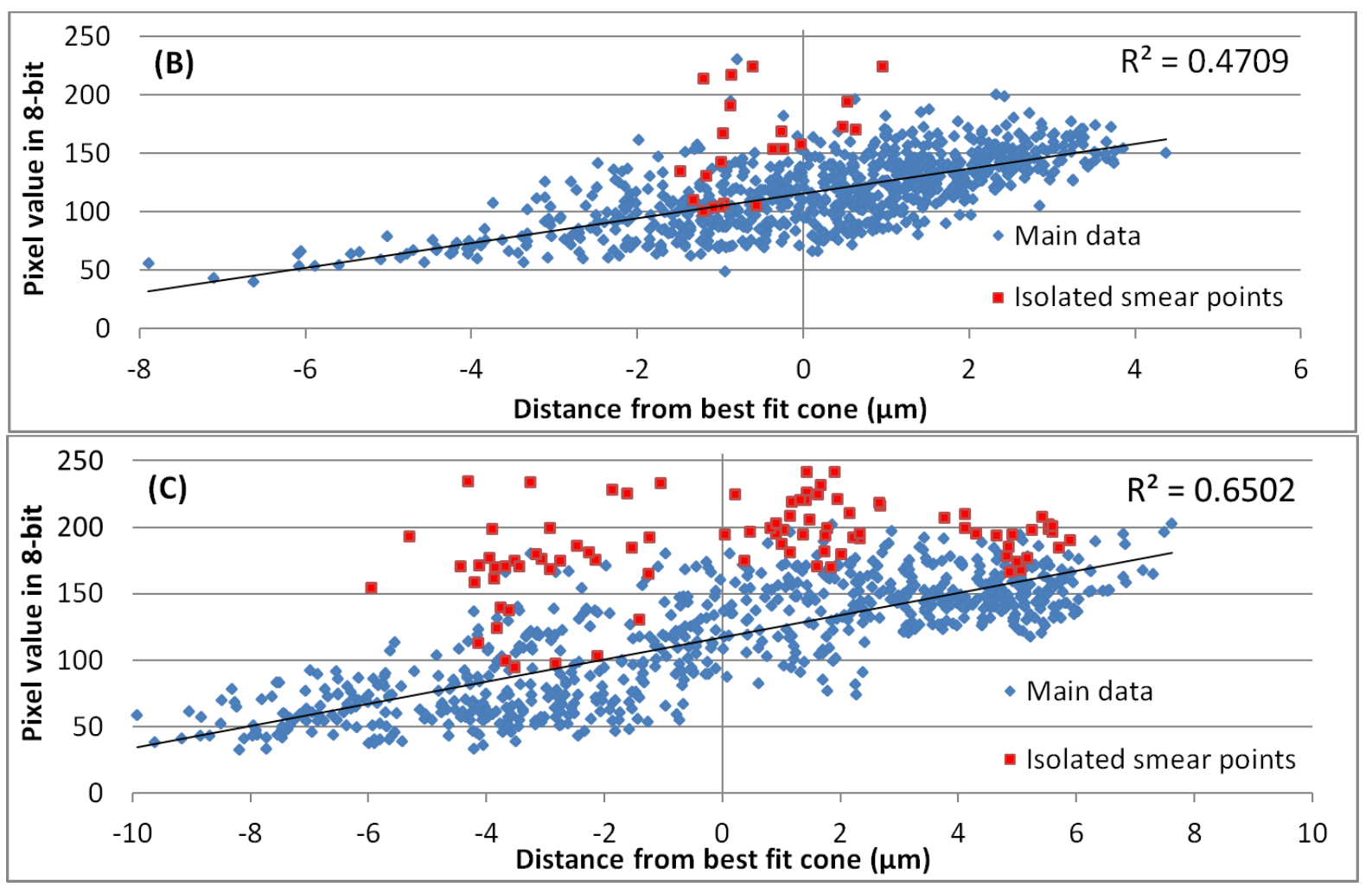

Figure 8: Pixel value against scanned distance from best-fit cone reduced to $32 \times 32$ shared grid-space for holes with 'Good' (A), 'Medium' (B) and 'Bad' (C) quality

These scatter plots allowed for a comparison of whether it was more effective to combine the three RGB (red, green, blue) channels into a single grey intensity, or if a single channel should be selected. As evidenced by the coefficients of determination in Table 1 there was a stronger relationship between the pixel value and the underlying geometry when using solely the red channel. This makes sense since the 'contact' regions of the image vary from blue to white and will thus have high pixel values over most of the image. This can be seen when comparing the two histograms as in Figure 9.

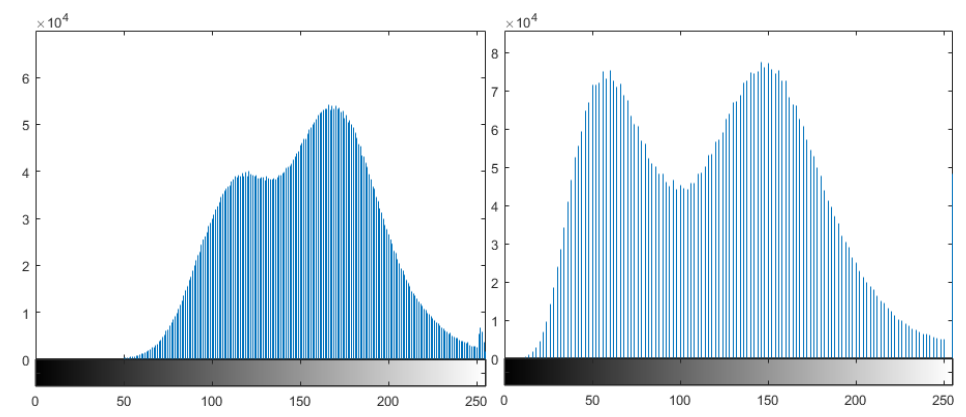

Figure 9: Pixel value histogram for combined greyscale image (left) and red channel (right) from 'Bad' combined image

The stronger peak separation in the right histogram further supports the use of the red channel for thresholding operations and general analysis by highlighting its effectiveness at separating the distinct areas. Of the three sets $\mathrm{R}^{2}$ increases with the images' standard deviation, as seen by the values in Table 1 . This could suggest that in images with significant variation between 
light 'contact' and dark 'non-contact' areas the colour is a better predictor of the geometry. It could also be a natural consequence of the geometric features which make one colour distribution more likely.

Table 1: Image analysis values

\begin{tabular}{ccccc}
\hline Image & Channel & Mean pixel & Pixel standard deviation & R-squared \\
\hline 'Good' & RGB & 134.2 & 27.2 & 0.3728 \\
\hline 'Medium' & RGB & 151.7 & 30.1 & 0.4147 \\
\hline 'Bad' & RGB & 152.5 & 38.3 & 0.5643 \\
\hline 'Good' & $\mathrm{R}$ & 95.9 & 34.8 & 0.4011 \\
\hline 'Medium' & $\mathrm{R}$ & 117.9 & 40.8 & 0.4423 \\
\hline 'Bad' & $\mathrm{R}$ & 119.4 & 53.8 & 0.6502
\end{tabular}

The three scatter plots do tend to show a positive correlation between the pixel value, and the displacement from best-fit cone. This is encouraging since it supports the hypothesis that the current method for inspecting Taper-lok fastener holes does measure what it sets out to. The plots do however still contain a reasonable amount of noise as seen by the values of $\mathrm{R}^{2}$. If the shade of the blue check perfectly indicated the displacement from the best-fit cone these three scatter plots would follow a single line. As they do not, the question must be raised as to whether this is an issue with the methodology, or a result of the mechanisms within the inspection. Evidence of the latter could identify limitations of using the blue check for inspecting Taper-lok holes.

A number of potential sources of noise are worth considering. The methodology of direct region-to-region comparison requires accurate alignment in order to be robust. While the two models' feature appear visually to overlap closely there is not as of yet a way to verify the spatial representation of a compilation image; this is considered beyond the scope of this research. The compilation images might also suffer from variations in illumination which could in turn vary local intensity values beyond their light absorptive properties. Further the light absorptive properties of engineer's blue are unknown, thus some of the noise may be associated with a non-linear change in shade with layer thickness increase.

Another potential issue the experiment identified is associated with maintaining the engineer's blue integrity during gauge removal. In comparing the data sets it can be observed 
that vertical scratches may seem longer than they actually are, likely due to the small pocket of blue filling the crack smearing during gauge removal. The inspection method does not have any way to compensate for the amount of blue remaining inside the sample hole during inspection. In this way it is unlikely to be effective at identifying small or deep features. It also can be observed to not maintain complete clarity in capturing horizontal machining marks; again this is likely due to shear forces during gauge removal.

Since this research aimed to investigate the effectiveness of the current method for Taper-lok inspection, the noise levels associated with the comparison could raise questions as to the method's effectiveness.

A key objective of this research was to investigate if a gauge imprint image's intensity directly relates to the gap size during gauge impact. However, since this size is impractical to measure the comparison is instead made to the unloaded bore geometry. This creates another potential source of noise as the blue imprint results from the bore geometry during impact which may be elastically deformed when compared to the unloaded geometries. It would have been preferable to record a loaded case gap size however attempts to CT scan test pieces could not reach useful resolutions due to the hardened steel gauge density, and the test piece size.

\section{$\underline{\text { Experimental results - Thresholding and deformation }}$}

From the scatter plot noise levels discussed in the previous section it becomes apparent that during Taper-lok inspection via blue check it is not entirely reliable to assume the shade of the blue indicates the impact zone geometries. This does not necessarily negate the effectiveness of the inspection however since this inspection does not currently distinguish by shades. Instead the inspector will visually assess regions as contact areas and non-contact areas acting like a visual thresholding. For this reason this second portion of data analysis evaluates the effectiveness of thresholding as a method to separate high and low contact areas.

In this thresholding experiment the grid-space was changed to $64 \times 64$. This finer grid size was chosen since this experiment aims to identify specific region in which thresholding is least effective as a means to identify contact. This brings the size of the averaged comparison regions closer to the size of the small features visibly identifiable in the images while still allowing some averaging to help alignment. 
First the red channel intensity image was thresholded using Otsu's method to minimise within-class variance and maximise between-class variance. Secondly the best-fit cone displacement data was thresholded to minimise discrepancy area. Regions not common to the thresholds are highlighted in red, see Figure 10. By fixing the intensity threshold and varying the displacement threshold this effectively identified the regions where thresholding is least effective as a means to identify the underlying geometry, and hence the geometric regions and features least suitable to being represented via the current Taper-lok inspection method. Since the various contact areas lead to different Otsu thresholding values the value from the 'Bad' image of 110/255 was used in all three, selected for its relatively distinct contact and non-contact regions with fairly similar areas.

(A)

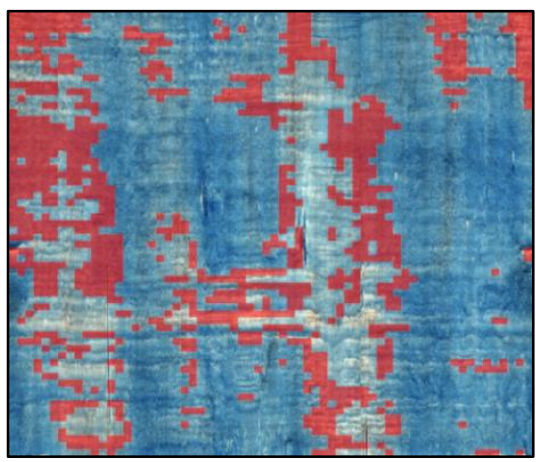

(B)

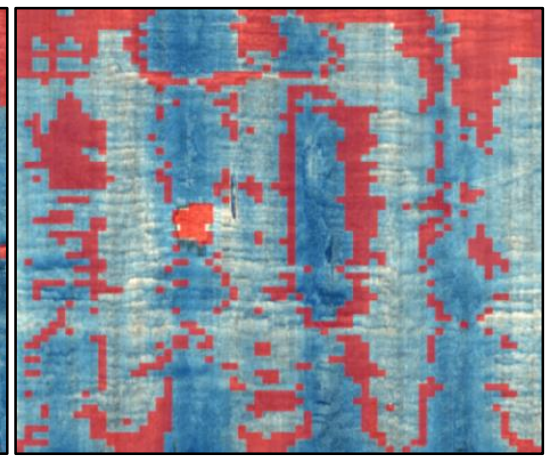

(C)

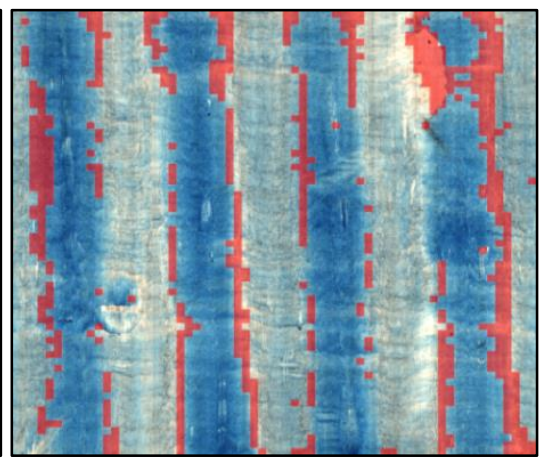

Figure 10: Regions of discrepancy between thresholds for holes with 'Good' (A), 'Medium' (B) and 'Bad' (C) quality

It can be seen from visual inspection that most of the regions in red exist at the boundaries between dark and light portions of the image. This is particularly noticeable in Figure 11 (C) where the red closely follows the edges of the leak paths. This is encouraging for supporting the inspection method in general since it suggests that for the majority of the image the separation into 'contact' and 'non-contact' is representative of the underlying geometry. Some issue may however still exist in the spatial representation of these regions. This could result from image creation or from issues with the inspection method itself. The percentage of the image with nonmatching threshold values makes up respectively $24.2 \%, 27.4 \%$ and $15.3 \%$ of the 'Good', 'Medium', and 'Bad' images.

In all three images the red discrepancy regions tend to be along light and dark boundaries featuring mainly on the bluer low-contact side suggesting the tendency is to under-identify contact area, instead of over-identify. If true this might suggest the inspection is more likely to create a false fail than a false pass; a lower consequence outcome. This could also indicate 
that a certain level of contact force is required to push the blue away that is only present in areas of strong contact. It could also result from a limit to the surface's elastic deformation.

(A)

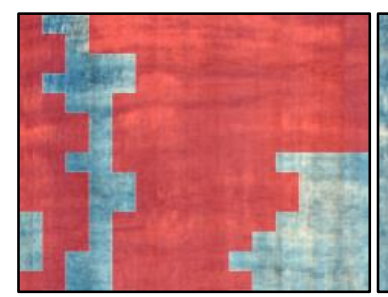

(B)

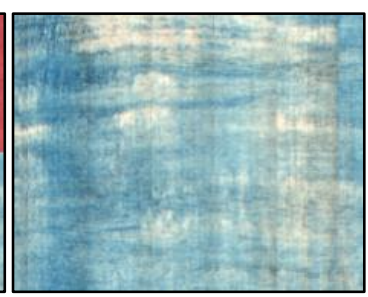

(c)

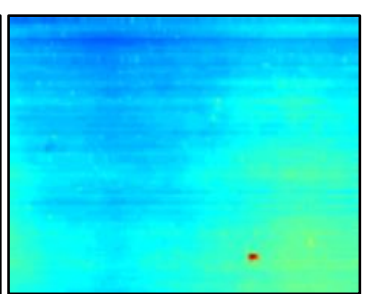

Figure 11: Comparison region with high discrepancy from 'Medium' bore hole from discrepancy (A), compiled (B), and radial distance $(\mathrm{C})$ images

There is an observable tendency in Figure 10 (A) and (B) for contact vertical paths to extend beyond where the unloaded data would suggest they stop, as illustrated in Figure 11. In this sectional comparison a large discrepancy area is seen with the inspection identifying it as contact area, but the radial values showing areas in blue, implying that these are below the thresholding value. This and similar examples could indicate that areas in contact are determined not only by unloaded geometry but also the amount of other supporting contact in its proximity. This would support the theory of local elastic deformation during impact. While the Taper-lok fastener relies on deformation for security it was not previously confirmed that the inspection also reaches deforming forces. By considering the extension of the contact regions horizontally into non-contact regions, and vertically to form continuous contact paths it would appear that deformation occurs more heavily along the vertical axis; the direction of forced movement.

If it is assumed that the Otsu thresholding value from the 'Bad' quality image forms a reasonable separation into contact/non-contact, then by considering the distances between the best-fit cone and the radial value threshold an estimate for the amount of elastic deformation occurring can be acquired. The values given in Table 2 give the radial mean and range for distances in 'uncontested' contact regions.

Table 2: Radial distances from reduced $64 \times 64$ grid-spaces to threshold value which minimises variation with thresholded compilation image

\begin{tabular}{cccc}
\hline Radial distance from threshold & Good & Medium & Bad \\
\hline Mean $(\mu \mathrm{m})$ & 2.0 & 2.6 & 1.7 \\
\hline Range $(\mu \mathrm{m})$ & 10.1 & 10.6 & 16.1 \\
\hline
\end{tabular}




\section{Conclusions}

Based on the experiments conducted and the respective coefficients of determination it seems reasonable to say that the shade of the engineer's blue, and in particular the red channel when imaged, is correlated to the underlying radial distance from a best-fit cone for the unloaded tapered bore geometry. Therefore it appears that the current method for inspecting Taper-lok holes is an appropriate method with which to identify areas of contact/non-contact and, thus, vertical leak paths with reasonable reliability.

Smearing and blue loss make the current method less reliable at identifying small features such as cracks or deep scratches, particularly horizontal ones which are transverse to the gauge's impact and removal axis. Smear points from removal are, unsurprisingly, unrepresentative of the underlying geometry and thus should be avoided wherever possible.

It should be noted that while thresholding, visually or by computer, may act as a useful tool for identifying contact regions, since a point's blue shade may be influenced by a combination of contact force, gap size and the surrounding contact levels, the threshold level does not strictly indicate the actual contact areas. The method is limited by its ability to remove engineer's blue from an area during impact. If there is nowhere to go then the blue will remain and the contact area may seem smaller than it truly is. For this reason the level of blue applied should ideally be controlled as the application of excessive amounts of engineer's blue will further exacerbate this issue.

\section{Bibliography}

Affatato, S. \& Ruggiero, A., 2019. Surface analysis on revised hip implants with stem taper for wear and failure incidence evaluation: A first investigation. Measurement, Volume 145, pp. $38-44$.

Bills, P. J., Racasan, R., Tessier, P. \& Blunt, L. A., 2015. Assessing the material loss of the modular taper interface in retrieved metal-on-metal hip replacements. Surface Topography: Metrology and Properties, Volume 3.

Bills, P. et al., 2017. Comparative study of material loss at the taper interface in retrieved metal-on-polyethylene and metal-on-metal femoral components from a single manufacturer. Engineering in Medicine, pp. 683 - 690.

Bishop, N. et al., 2013. Wear Patterns of Taper Connections in Retrieved Large Diameter Metal-on-Metal Bearings. JOURNAL OF ORTHOPAEDIC RESEARCH, pp. 1116 - 1122. 
Buente, D., Huber, G., Bishop, N. \& Morlock, M., 2015. Quantification of material loss from the neck piece taper junctions of a bimodular primary hip prosthesis. A retrieval study from 27 failed Rejuvenate bimodular hip arthroplasties. The Bone and Joint Journal, pp. 1350 1357.

Clark, G., 2005. A Review of Australian and New Zealand Investigations on Aeronautical Fatigue During the Period April 2003 to March 2005, s.l.: Commonwealth of Australia 2005.

Harding, C., Hugo, G. \& Bowles, S., 2006. Automated Ultrasonic Inspection For Crack Detection At F-111 Lower Wing Skin Fastener Holes. Auckland, New Zealand, A-PCNDT.

He, G., Liu, P., Guo, L. \& Wang, K., 2014. Conicity error evaluation using sequential quadratic programming algorithm. Precision Engineering, 38(2), pp. 330 - 336.

Hothi, H. S. et al., 2017. Effect of Bearing Type on Taper Material Loss in Hips From 1 Manufacturer. The Journal of Arthoplasty, pp. 1588 - 1593.

Hothi, H. S. et al., 2013. The Reliability of a Scoring System for Corrosion and Fretting, and Its Relationship to Material Loss of Tapered, Modular Junctions of Retrieved Hip Implants. The Journal of Arthroplasty, pp. 1313 - 1317.

Lambert, W. O., 1974. Bearing Check Gauge. United States, Patent No. 3,829,977.

Martin, A. J., Jenkins, D. R. \& Citters, D. W. V., 2018. Role of Corrosion in Taper Failure and Head Disassociation in Total Hip Arthroplasty of a Single Design. Journal of Orthopaedic Research, pp. 2996 - 3003.

Matthies, A. K. et al., 2013. Material Loss at the Taper Junction of Retrieved Large Head Metal-on-Metal Total Hip Replacements. Journal of Orthopaedic Research, pp. 1677 - 1685.

Molent, L., 2010. Fatigue crack growth from flaws in combat aircraft. International Journal of Fatigue, 3 September, Volume 32, pp. 639 - 649.

Morlock, M. M. et al., 2006. Biomechanical, morphological, and histological analysis of early failures in hip resurfacing arthroplasty. Engineering in Medicine, Volume 220, pp. 333 344.

Salter, L., Estates, P. V. \& Briles, F. S., 1966. Method of prestressed fastening of materials. United States, Patent No. 3,270,410.

Tsoi, K. A. \& Rajic, N., 2008. Interference fit fastener inspection using sonic thermography. Australian Journal of Mechanical Engineering, 6(2), pp. 113 - 118. 